\title{
Bioinformatic identification of cassava miRNAs differentially expressed in response to infection by Xanthomonas axonopodis pv. manihotis
}

\author{
Álvaro L Pérez-Quintero, Andrés Quintero, Oscar Urrego, Pablo Vanegas and Camilo López
}

\begin{abstract}
Background: microRNAs (miRNAs) are short RNA molecules that control gene expression by silencing complementary mRNA. They play a crucial role in stress response in plants, including biotic stress. Some miRNAs are known to respond to bacterial infection in Arabidopsis thaliana but it is currently unknown whether these responses are conserved in other plants and whether novel species-specific miRNAs could have a role in defense.

Results: This work addresses the role of miRNAs in the Manihot esculenta (cassava)-Xanthomonas axonopodis pv. manihotis (Xam) interaction. Next-generation sequencing was used for analyzing small RNA libraries from cassava tissue infected and non-infected with Xam. A full repertoire of cassava miRNAs was characterized, which included 56 conserved families and 12 novel cassava-specific families. Endogenous targets were predicted in the cassava genome for many miRNA families. Some miRNA families' expression was increased in response to bacterial infection, including miRNAs known to mediate defense by targeting auxin-responding factors as well as some cassava-specific miRNAs. Some bacteria-repressed miRNAs included families involved in copper regulation as well as families targeting disease resistance genes. Putative transcription factor binding sites (TFBS) were identified in the MIRNA genes promoter region and compared to promoter regions in miRNA target genes and protein coding genes, revealing differences between MIRNA gene transcriptional regulation and other genes.
\end{abstract}

Conclusions: Taken together these results suggest that miRNAs in cassava play a role in defense against Xam, and that the mechanism is similar to what's known in Arabidopsis and involves some of the same families.

\section{Background}

Very succinctly plant-bacteria interactions can be thought as governed at molecular level mainly by three types of proteins: plant PRRs (pathogen recognition receptors), bacterial effectors and plant resistance proteins. PRRs are proteins recognizing highly conserved structures and molecules in microorganisms named MAMP (microbial-associated molecular patterns) and mediate MAMP-triggered immunity (MTI), which is efficient against non-adapted pathogens. Pathogens have developed effector proteins to suppress MTI. In turn, plants can counteract the action of effector by the specific recognition of effectors mediated by resistance proteins which will trigger a strong defence response known as ETI (effector-triggered immunity) [1].

\footnotetext{
* Correspondence: celopezc@unal.edu.co
}

Biology Department, Universidad Nacional de Colombia, Calle 45 Cra 30, Bogota, Bogota DC, Colombia
During the past decade, small RNAs have also been found to be key players in mediating plant-pathogen interactions as well as many other biological processes. microRNAs (miRNAs) are important regulators of eukaryotic gene expression. They are transcribed from nuclear MIRNA genes by RNA polymerase II (RNA pol II) into primary miRNAs (pri-miRNAs). The pri-miRNAs are then processed in plants by Dicer-like proteins (DCL) into precursor miRNAs (pre-miRNAs) which form a characteristic hairpin-like structure [2,3]. A subsequent processing step by DCL slices the pre-miRNA to form a miRNA:miRNA* duplex (21-22 nt). The duplex is then methylated and exported from the nucleus to the cytoplasm where it is recognized by an argonaute (AGO) protein and incorporated into the RNA-induced silencing complex (RISC). Only the mature miRNA strand (usually the one having less stable 5 ' end pairing) is retained in the complex, while 
the passenger (miRNA*) strand becomes degraded [3]. However, in some cases, miRNA* has been detected as being expressed at the same or even at higher levels than the leader strand and may have silencing activity [4]. The RISC complex will guide complementary mRNA (targets) silencing, usually by cleavage between the $10^{\text {th }}$ and $11^{\text {th }}$ nt of the paired miRNA [3].

An early miRNA pathway control mechanism comes from MIRNA gene transcription regulation by cis-regulatory elements and trans-acting factors. Recent works have attempted to identify key elements involved in miRNA regulation [5-9]; however, little is yet known about miRNA co-regulation under different conditions and the mechanisms involved.

Most known plant miRNAs target transcription factors which play an important role in regulating plant development. There is now increasing evidence of miRNA's importance in response to biotic and abiotic stress in plants $[2,10]$. Reprogrammed miRNA-mediated gene expression during plant immune response has not been studied in depth, but is potentially an important element for controlling pathogen invasion. It has been demonstrated that bacteria-induced miR393 mediates anti-bacterial defense of $A$. thaliana against Pseudomonas syringae pv. tomato (Pst) by targeting TIR1, an F-box family of auxin receptors and consequently repressing auxin signaling [11]. In turn, bacteria use effector proteins to disrupt miRNA accumulation [12]. The repertoire of known bacterial-responsive miRNAs has increased and includes several families known to regulate hormone signaling, such as miR160, miR167 and miR390 involved in auxin signaling, miR159 involved in ABA signaling and miR319 involved in jasmonic acid signaling [13-15].

Cassava (Manihot esculenta) is a staple crop which stores important quantities of starch in its roots. These roots constitute the main source of calories for more than half a billion people around the world, mainly in tropical regions [16]. The starch also has important uses in industry, including bioethanol production [16-18]. Cassava has remarkable tolerance to abiotic stress, it can be cultivated in low-fertility acidic soils and is highly tolerant to drought [16]. Its production can be severely affected by cassava bacterial blight (CBB), caused by gram-negative bacteria Xanthomonas axonopodis pv. manihotis $(\mathrm{Xam})$. This disease is present in all regions where cassava is grown and production losses can reach up to $80 \%$ or $100 \%$ [19]. CBB incidence, as that of many plant diseases, is expected to increase greatly with climate change [20]. This, along with the increasing human population, makes it essential to understand the underlying mechanism of plant antibacterial defense, aimed at producing biotechnological strategies for crop genetic improvement.
The cassava miRNA repertoire is mostly unknown. Up to 20 conserved miRNA families have been indentified in ESTs collections by using bioinformatics approaches [21-23] and the expression of 23 mature miRNA families in cassava and other euphorbiaceous has been studied [24]. However no miRNAs from cassava are currently deposited in miRBase, the consensus database for verified miRNAs [25]. The first draft of the cassava genome was released in October 2009 and a new version was made available in October 2010 [26]. This is an important tool for the discovery and prediction of new and specific cassava miRNAs.

This study characterizes the cassava miRNA repertoire using expression data from small RNA libraries and identifies pre-miRNAs in the cassava genome. The miRNA-mediated response to Xam infection in cassava is also analyzed as well as possible transcription factors involved in miRNA regulation.

\section{Results}

\section{Deep sequencing of cassava sRNA libraries}

Small RNA profiling libraries sequenced with Illumina SBS technology were used to study the role of cassava miRNAs in response to Xam. Two small RNA libraries were constructed using RNA extracted from leaves and stems from the Xam-resistant cassava variety MBRA685. One of them was not inoculated (NI) and the other inoculated (I) with the highly aggressive Xam strain CIO151. The inoculated library was constructed from an RNA pool of various post-inoculation times so only robust and consistent responses could be detected. 15 and 11 million reads trimmed reads (adapters removed) were obtained from the NI and I libraries, respectively (Table 1). Most trimmed reads in both libraries were in the 20-24nt range (most small RNA sizes) (Figure 1a). Processed and raw files for these libraries can be accessed at the NCBI Gene Expression Omnibus under accession number GSE29379.

Reads from both libraries were mapped to the cassava genome (Table 1), resulting in eight million (NI) and seven million (I) reads perfectly mapping to the genome. Reads mapping to known snoRNAs, tRNAs, and rRNAs were then removed and 5,655,383 and 5,187,027 reads were obtained from the NI and I libraries, respectively, and used for further analysis.

Both libraries were also mapped against the Xam genome to verify the presence of nucleic acids from bacteria in the I but not in the NI library (Table 1). As expected, fewer reads from the NI library mapped the Xam genome $(0.8 \%)$ compared to the I library (5.2\%). Most NI reads matching the Xam genome also matched the cassava genome (91\%) and were thus shared short regions between both genomes. Only $33 \%$ of the I reads mapping to the Xam genome corresponded to these shared 
Table 1 General statistics for cassava small RNA library processing and mapping

\begin{tabular}{lll}
\hline & Non-inoculated reads & Inoculated reads \\
\hline Total & $31,434,907$ & $15,968,516$ \\
\hline High quality, adaptor-trimmed & $15,084,481$ & $11,871,330$ \\
\hline Matching cassava genome & $8,844,221$ & $7,226,346$ \\
\hline Matching cassava genome, Non-redundant $(\mathrm{nr})$ & $1,183,868$ & 653,480 \\
\hline Not matching rRNAs, snoRNAs, tRNAs* & $5,187,027$ \\
\hline Not matching rRNAs, snoRNAs, tRNAs (nr)+ & $5,655,383$ & $1,664,361$ \\
\hline Matching Xam genome & $2,906,014$ & $298,811(5.2 \%)$ \\
\hline Matching Xam genome (nr) & $45,416(0.8 \%)$ & $80,850(4.8 \%)$ \\
\hline Matching known miRNAs & $6,952(0.2 \%)$ & $122,273(2.3 \%)$ \\
\hline Matching known miRNAs (nr) & $42,356(0.7 \%)$ & $3,398(0.2 \%)$ \\
\hline Known miRNA families & $4,921(0.1 \%)$ & 48 \\
\hline Matching cassava intergenic regions & 53 & $599,178(11.5 \%)$ \\
\hline Matching cassava intergenic regions (nr) & $522,080(9.2 \%)$ & $64,536(3.8 \%)$ \\
\hline
\end{tabular}

*Percentages and normalized values throughout the work were calculated on these total reads. +percentage for non-redundant reads were calculated based on these total reads

regions; the remaining $67 \%$ may represent non-coding RNA or degraded RNA from Xam.

\section{miRNA and pre-miRNA identification}

To identify conserved miRNAs, reads from both libraries were mapped to a set of known plant miRNAs from miRBase [25] and PMRD [27]; 56 conserved miRNA families were identified in cassava. Reads mapping to known miRNA were predominantly $21 \mathrm{nt}$ long (Figure 1b). These miRNAs were also screened using miRProf [28] and all 56 families were confirmed with this method. Unlike miRBase, PMRD includes miRNAs without experimental validation. Only seven PMRDexclusive miRNA families mapped against small RNA cassava libraries; these were taken into account for premiRNA identification but no precursor could be identified in the cassava genome and were excluded from later analysis.

Reads for the 56 conserved miRNAs families were mapped against the genome and 485 adjacent regions were analyzed finding that 116 met the main criteria for being considered as real pre-miRNAs (structural features and secondary structure statistical test). These pre-miRNAs represented 24 miRNA families (Additional file 1). pre-miRNAs for some families were not found which could be explained by considering the cassava genome incomplete sequencing or the presence of non-canonical pre-miRNAs.

To identify novel miRNAs, reads ranging from 20 to $24 \mathrm{nt}$ from both libraries were mapped against cassava intergenic regions (Table 1 ) and the adjacent \pm 150 nt region was extracted (in total 64,876 regions). miRchek [29], miPred [30] and miReap [31] were used for initial filtering of possible pre-miRNAs. Candidates meeting the main criteria mentioned above (structural features and secondary structure statistical test), as well as having supporting evidence from miRcheck, miReap or miPred, were considered to be real pre-miRNAs. Twelve new miRNA precursors from 12 miRNA families were identified and named Cass_miRA through Cass_miRL (temporary names) (Additional file 1).

Reads mapping to the predicted novel miRNAs were mostly 21 nt long, as was observed for conserved miRNAs (Figure 1c). A Blastn [32] search of Genbank's nucleotide $\mathrm{nr}$ database for high pre-miRNA conservation in other plant genomes revealed no similarity (e-value < $0.001,>80 \%$ coverage) with plant nucleotide sequences different from cassava in 10 out of the 12 pre-miRNA families, whereas two pre-miRNAs, Cass-miRK and Cass_miRL, resemble non-coding sequences from Populus thricocarpa and Helianthus petiolari respectively.

\section{Target prediction}

Targets were predicted for all miRNAs identified among annotated genes in the cassava genome using a modified version of miRanda software [33]; 277 possible targets for 43 conserved miRNA families were identified as well as 70 targets for the 12 new miRNA families (Additional file 2). Targets were also searched for all miRNAs in the cassava genome using psRNAtarget http://plantgrn.noble.org/ psRNATarget/. Additional support as real targets was obtained for 162 out of the 347 targets identified with miRanda (Additional file 2). A high percentage of possible targets identified were transcription factors $(24 \%)$, as is common for many known plant miRNAs [2]. Other commonly predicted targets were kinases (7\%), DNA binding proteins $(3 \%)$ and disease resistance proteins (3\%). Four families (miR167, miR397, miR894 and Cass_miRJ) had 


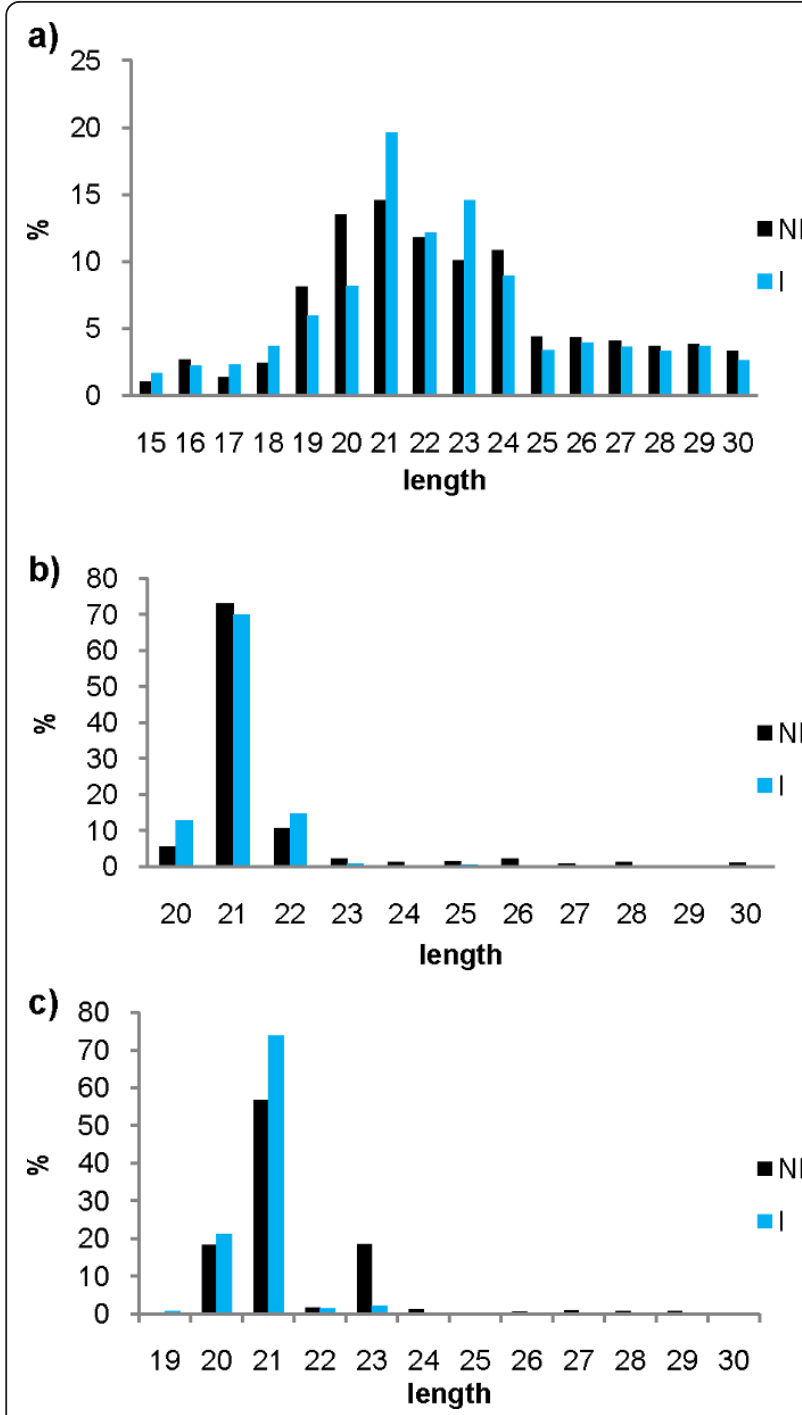

Figure 1 Size frequencies of trimmed and miRNA-mapped sRNA reads from inoculated and not inoculated cassava

libraries. a) Size frequencies of trimmed unmapped reads in both libraries. b) Size frequencies of reads in both libraries mapping to known plant miRNAs. c) Size frequencies of reads in both libraries mapping to new cassava miRNAs.

among their possible targets genes involved in starch biosynthesis or metabolism, as identified by similarity with genes in the KEGG pathway (map00500), which could be important focus points for biotechnological strategies aiming at bioethanol production.

\section{miRNA differential expression}

Cassava's miRNAs expression levels were quantified as normalized reads mapping to each mature miRNA in both libraries. 10 conserved miRNA families had highly increased expression $\left(\log _{2}\right.$ fold $\left.>2\right)$ in response to Xam infection (Figure 2a), including miR160 and miR167 a)

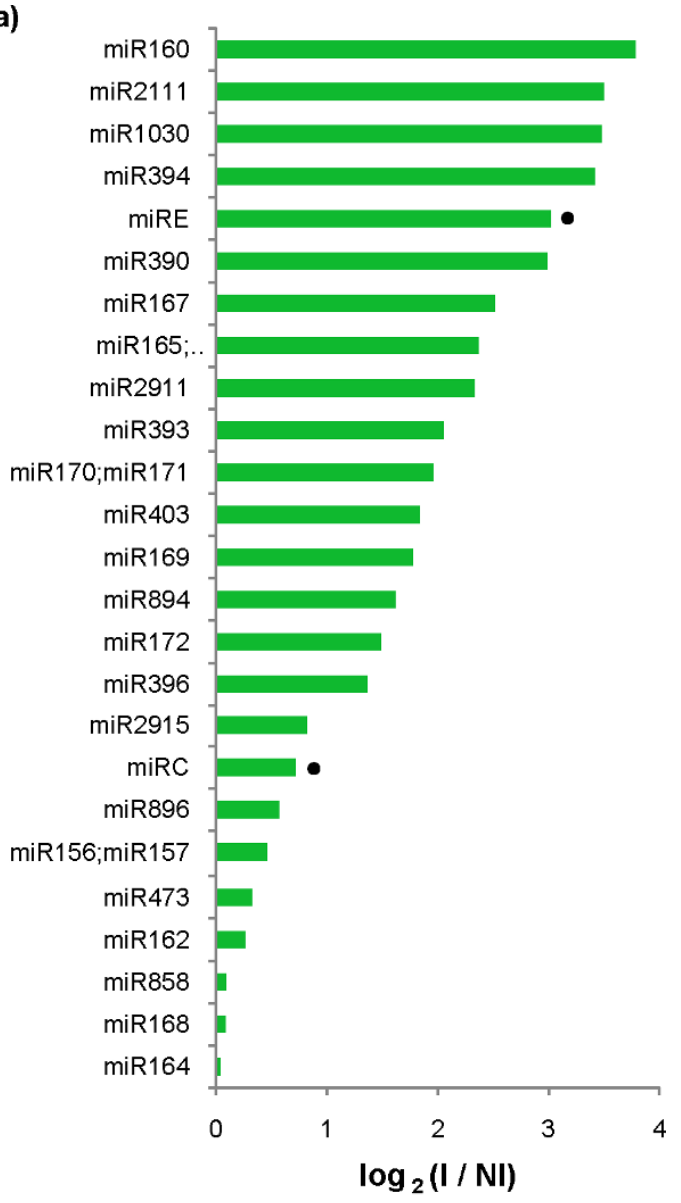

b)

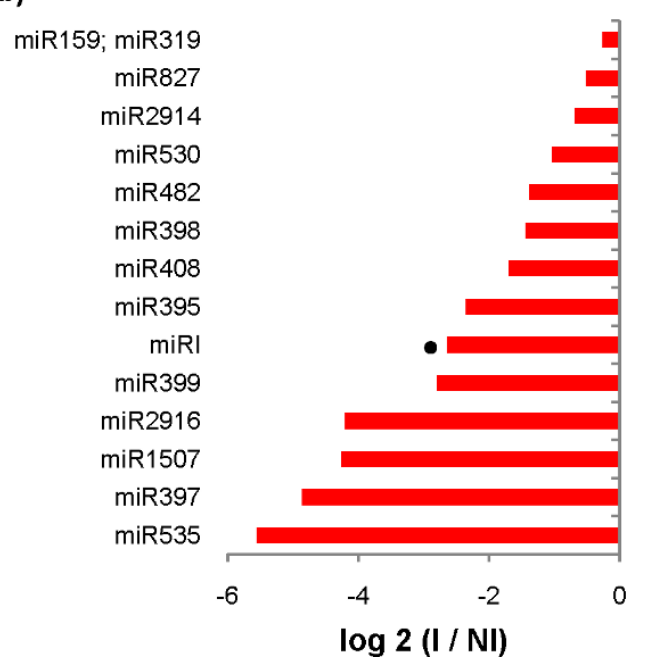

Figure 2 Differential expression of miRNA families in response to bacterial infection. a) Bacteria-induced miRNAs. b) Bacteriarepressed miRNAs. Only families having expression values greater than 10 normalized reads in at least one small RNA library are shown. Black circles indicate novel cassava miRNAs. 
families which are both known to target auxin response factors (ARFs) [13] and miR393 and miR390 families which are also known to regulate auxin signaling [13]. Predicted targets for miR160 in cassava were ARF-like genes whereas predicted targets for miR167 included phosphatases and peptidases. These targets' downregulation in response to bacteria was confirmed through semi-quantitative RT-PCR (Figure 3). It was possible to confirm predicted target downregulation for other induced conserved miRNA families, including miR394, miR165 and miR171 which target an F-box family protein, an ATHB transcription factor and a scarecrow-like transcription factor, respectively (Figure 3). In some of these cases (miR171, miRE, miR394, miR197b) target expression decreased at 4 days post-inoculation (dpi) and then increased at $8 \mathrm{dpi}$, but never reached higher expression values than those without inoculation. This can be expected since libraries were constructed from an RNA pooled from different times post inoculation. In spite of the importance of these data, a time specific quantification of mature miRNAs is still needed to make an accurate correlation with target expression.

Induced miRNAs
Target miR160 (4.1_002668m)
Target miR394 (4.1_008769m)
Target miR167a (4.1_015606m)
Target miR167b (4.1_009942m)
Target miR165 (4.1_001674m)
Target miR171 (4.1_002034m)
Target miRE (4.1_002016m)
Repressed miRNAs
Targure 3 miRNA target expression shown by semi-quantitative
leaves inoculated with Xam CIO151collected at 0 days post
inoculation (dpi), 4 dpi and 8 dpi. Gene names from the cassava
genome annotation appear in parenthesis.
Target miR408 (4.1_019179m)

On the other hand seven miRNA families' expression became reduced in response to $\mathrm{Xam}$ infection ( $\log _{2}$ fold $<-2$ ) (Figure 2b). These repressed families included miR535, miR395 and miR482 which were predicted to target various candidate NB-LRR and LRR disease resistance proteins. The miR397, miR398 and miR408 families were also repressed $\left(\log _{2}\right.$ fold $\left.<-1.4\right)$; they are involved in copper regulation by targeting laccases, copper superoxide dismutases and plantacyanins, respectively [34]. It was possible to confirm bacteria-induced increased expression for a resistance-like protein predicted to be targeted by miR535 (induced at $4 \mathrm{dpi}$ ) and for a plantacyanin predicted to be targeted by miR408 (induced at $8 \mathrm{dpi}$ ) (Figure 3 ).

Novel miRNAs had overall low expression levels. Only three novel miRNAs (Cass_miRC, Cass_mirE and Cass_miRI) had expression values higher than 10 normalized reads in at least one library. Thus, while many showed differential expression in response to bacteria they were not taken into account due to their low expression values. Species-specific miRNAs are known to have low expression compared to conserved miRNAs [35]. Cass_miRE was highly induced in response to bacteria $\left(\log _{2-}\right.$ fold $=3$ ) (Figure 2a) and downregulation of its predicted target, a kinase related to $A$. thaliana FERONIA, was determined by RT-PCR (Figure 3). This gene is known to be involved in pathogen interactions and pollen development [36,37].

The passenger strand (miRNA*) becomes rapidly degraded after miRNA:miRNA* duplex disruption and leader strand incorporation into RISC [3]. Its expression can nevertheless be detected in small RNA libraries and it is considered supporting criteria for miRNA validation [38]. miRNA* expression for cassava miRNAs was quantified as the number of reads in each library strand-specifically mapping to cassava miRNAs* predicted from pre-miRNAs. miRNA* expression was detected for 11 conserved miRNA families. All families had miRNA/ miRNA* ratios higher than 1 in both NI and I libraries, meaning that as expected the leader strand was always more highly expressed than the passenger strand. The miRNA/miRNA* ratio changed for each miRNA family between NI and I library (Figure 4a). A change in miRNA/miRNA* ratio may have indicated differential degradation of the miRNA* strand in different conditions; however, it was most likely a stochastic effect due to the detection of degrading molecules.

It is thought that miRNA silencing signal can be amplified by the production of transient siRNAs derived from cleaved target mRNAs [39]. Reads from both libraries were specifically mapped to predicted cassava miRNA targets' sense or antisense strand to assess the extent to which transitivity or miRNA silencing signal amplification occurred in cassava. Reads mapping to the 


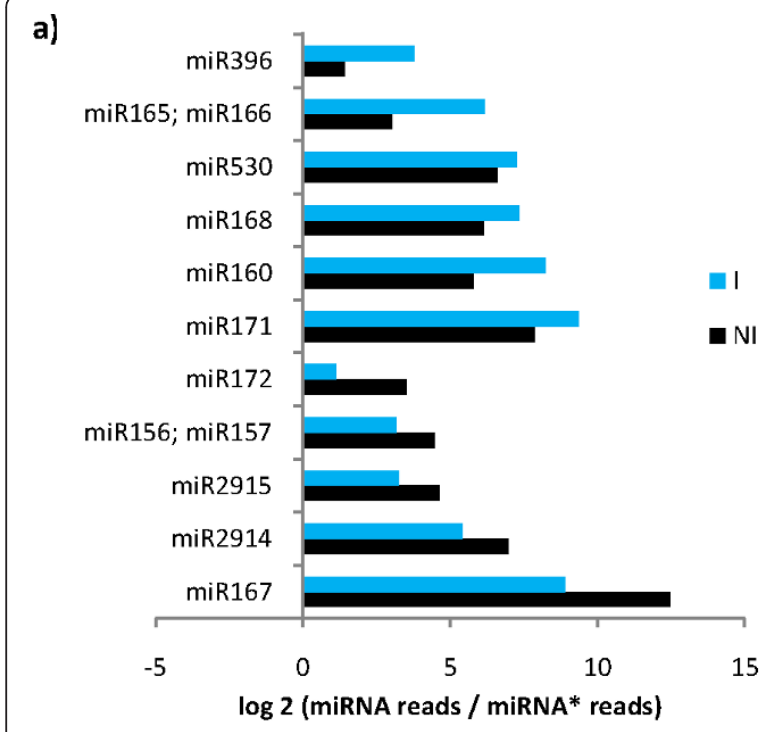

b)

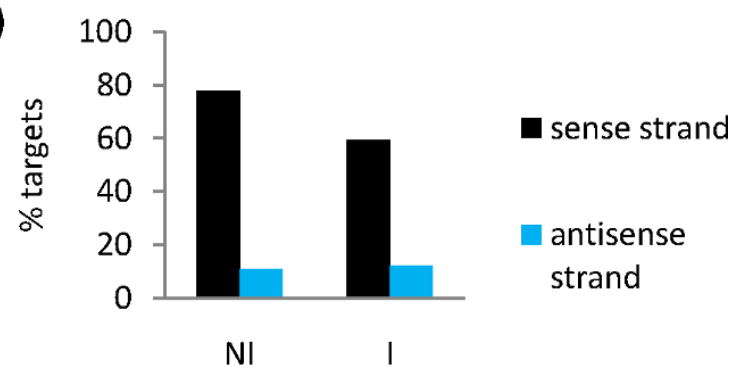

c)

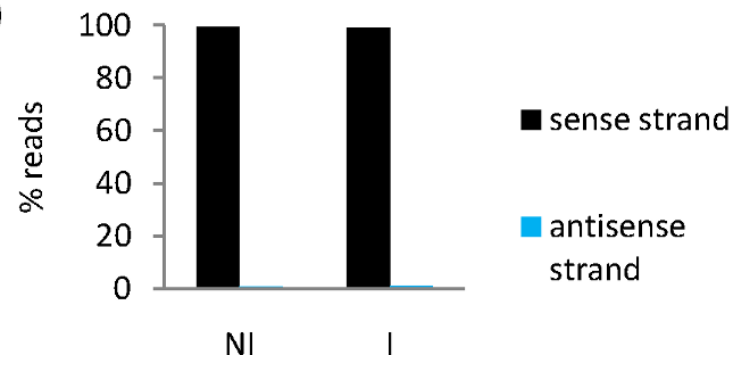

Figure 4 miRNA* expression and transitivity analysis. a) $\log 2$ miRNA/miRNA* ratio shown for families in which miRNA* expression was detected. b) Targets for which reads matching sense and antisense strand were detected in both libraries. c) Reads mapping to target mRNA sense and antisense strands in both libraries.

antisense strand would indicate the presence of dsRNAderived transitive siRNAs after RdRP recognition, while reads mapping to the sense strand are likely to be predominantly cleaved miRNA target products as well as possible siRNAs. The percentage of predicted miRNA targets with reads mapping to the antisense strand (targets producing transitive siRNAs) in both libraries was around $10 \%$ (Figure $4 \mathrm{~b}$ ). Reads mapping to miRNA targets' antisense strand (107 reads in NI, 235 reads in I) were around $1 \%$ of reads mapping to predicted targets (Figure 4c), i.e. possible transitive siRNA expression. This showed that only a small percentage of targeted genes produced transitive siRNAs and they were produced in very small amounts compared to miRNA target cleavage rate. These results suggest that miRNA signaling amplification through target transitivity is not a prevalent mechanism in cassava.

\section{Promoter analysis}

MIRNA genes are transcribed by RNA pol-II after recognition by transcription factors; however, little is known about miRNA transcriptional regulation [5]. Transcription factor binding sites (TFBS) were identified in the 1000nt promoter region upstream of identified cassava pre-miRNAs to find possible patterns in MIRNA gene regulation in response to bacteria. Binding sites for 59 transcription factors were identified in 116 miRNA promoter regions. Commonly found regulatory motifs included the TATA and GATA box and RAV (ABI3/ VP1-related) and LFY (Leafy) TFBS (Figure 5).

It has been found that ARF binding sites are overrepresented in auxin-related miRNA families like miR160 and miR167, thus forming a regulatory loop [40]. In our results miR160 was also the family having the most ARF binding sites identified (10 out of 44 ARF binding sites were found in miR160 promoter regions).

The same predictions were then made in promoter regions of cassava miRNA target genes and a set of 1,000 randomly-chosen cassava genes to determine whether miRNAs were differently transcribed from other groups of genes. Paired wilcoxon tests were made comparing the frequency distributions in each group finding significant differences $(\alpha=0.1)$ between MIRNA genes and randomly selected genes $(p$-value $=0.001248)$ and between MIRNA genes and miRNA targets ( $p$-value

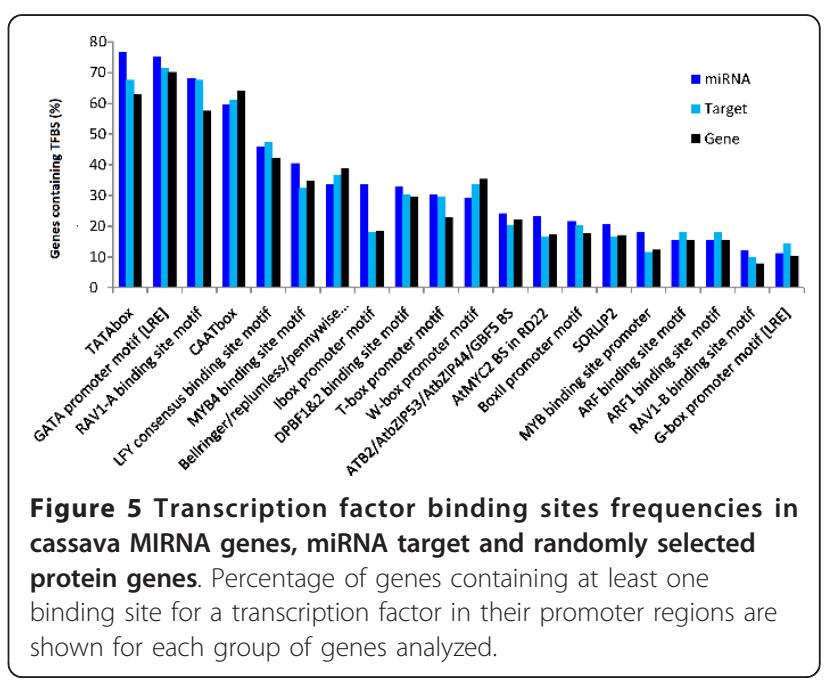


$=0.08904$ ), whereas no differences were found between target genes and randomly selected genes ( $p$-value $=$ 0.5462 ). However, no significant differences were found between the frequency distributions of miRNA families highly induced or repressed in response to bacteria and those of non-differentially expressed miRNA families $(p-$ value $>0.05$ ).

\section{Discussion}

A general model has been proposed based on Arabidopsis-Pseudomonas interaction results describing miRNAmediated response to bacterial infection. In this model miRNAs targeting negative defense response regulators are induced and miRNAs targeting positive regulators (e.g. resistance genes) are repressed upon bacterial infection $[41,42]$. In this work we reported the differential expression of some cassava miRNA families in response to Xanthomonas axonopodis pv. manihotis, generally agreeing with this model. miRNAs induction was found to be involved in regulating auxin signaling: miR160, miR167, miR390 and miR393 [11,13]. Conservation of these miRNA families' expression pattern in Arabidopsis and cassava pointed to a plant conserved defense mechanism, likely to be related to PTI, since some of these families have been shown to be induced in response to flagellin [14]. Thus, auxin signaling disruption seems to be an important strategy for impairing bacterial growth in plants. Recent work has shown that enzymatic disruption of auxin signaling is an important mechanism for broad-spectrum resistance and that pathogens secrete auxins during infection to render a plant vulnerable by loosening the cell wall $[43,44]$. Some conserved miRNA families not present in A. thaliana were induced expressed in response to Xam infection, including miR2911 and miR1030 families which have so far only been identified in Populus euphratica and Physcomithrella patens, respectively [45]. The role they might play in mediating plant-bacteria interactions remains unknown.

miRNA downregulation showed in this work also agrees with the general model, since three repressed families (miR535, miR395 and miR482) were predicted to target NB-LRR and LRR resistance-like genes in cassava. These genes' subsequent increased expression may have a role in cassava-specific ETI response; however, this still needs to be demonstrated. Other repressed families were miR397, miR398 and miR408, known to be involved in copper regulation and to be differentially expressed in response to biotic stress [34,46,47]. Copper is widely used as a pesticide in agriculture due to its antibacterial effect [48]. It has been shown that some Xanthomonas oryzae strains use transcription activatorlike (TAL) effector proteins to modify copper distribution in rice to render the plant susceptible [49].
miRNA-mediated regulation of copper homeostasis could also be crucial as a bacterial defense mechanism.

Transitivity was analyzed by quantifying possible target cleavage-derived transient siRNAs. This mechanism has been studied in detail for some specific cases in $A$. thaliana [50-52] and in the moss Physcomitrella patens [53]. A recent study has suggested, however, that the mechanism could be more widespread than once thought; analyzing next-generation sequencing of small RNA libraries, the same percentage was found for small 21nt reads mapped against Arabidopsis miRNA targets in both sense and antisense strands [39]. On the contrary, it was found in this work that reads mapping o cassava targets' antisense strand were greatly underrepresented in cassava libraries $(<1 \%)$ and only around $10 \%$ of predicted targets produced antisense reads, agreeing with transitivity being an infrequent mechanism.

Finally, TFBS were predicted on the promoter region of MIRNA genes finding that MIRNA genes appeared to have overall a different frequency distribution from those of protein coding genes including miRNA targets suggesting the presence of regulatory elements acting specifically on MIRNA genes. It has been previously reported that MIRNA genes are abundant in TATA-box, AtMYC, ARF, SORLEP3 and LFY binding sites, compared to protein encoding genes [40]. In our results the frequency distribution of TFBS in these groups of genes reveals that namely the TATA-box, MYB4 and L1-box motifs were more abundant in MIRNA genes. However, no differences in TFBS frequencies could be found between bacteria-induced or bacteria-repressed miRNA families and families that were not differentially, this suggests that miRNA transcriptional regulation in response to bacteria may be family-specific or locispecific.

\section{Conclusions}

This work has shown in-depth characterization of cassava miRNAs in response to Xam infection and has shed new light on these molecules' importance in plantpathogen interactions. These data are encouraging but still preliminary and further experimental validation is still needed to fully understand the impact of the miRNA pathway in the cassava-Xam interaction. Understanding miRNA regulation and/or that of their targets could (given miRNA pathway flexibility) lead to developing better biotechnological strategies aimed at producing cassava plants having enhanced resistance to pathogens.

\section{Methods}

Plant materials and plant inoculation

Cassava plants were grown from mature stem cuttings and kept in a greenhouse at $26-30^{\circ} \mathrm{C}$, with $12 \mathrm{~h}$ day- 
light photoperiod and $80 \%$ relative humidity. Cassava variety MBRA685 (resistant to Xam-CIO151) was used for small RNA library construction and variety MCOL1522 (susceptible to Xam-CIO151) was employed for RT-PCR experiments.

Six-week-old plants were inoculated with $36 \mathrm{~h}$-old cultures of the aggressive Xanthomonas axonopodis pv. manihotis strain CIO151 in both leaves and stems. Leaves were inoculated by piercing six holes in the mesophyll and placing a $5 \mu \mathrm{L}$ drop of a liquid Xam$\mathrm{MgCl}_{2}$ culture calibrated at $\mathrm{OD}_{600 \mathrm{~nm}}=0.002(1 \times$ $\left.10^{8} \mathrm{cfu} / \mathrm{ml}\right)$. Two leaflets per leaf and three leaves per plant were inoculated. Stems were inoculated by puncture. At least three plants per collection time were inoculated for each experiment.

\section{Small RNA library construction and sequencing}

For the inoculated library leaves and stems were collected from inoculated plants $(0 \mathrm{~h}$ post inoculation $-\mathrm{hpi}$, $6 \mathrm{hpi}, 24 \mathrm{hpi}, 2$ days post-inoculation -dpi, 5 dpi, 7 dpi and $15 \mathrm{dpi})$.. RNA extractions were made using a $\mathrm{LiCl}-$ acid phenol:chloroform method. RNA extractions from inoculated plants (at least six plants per time point) were pooled together in equal amounts. For the noninoculated library a single RNA extraction from tissues from six untreated plants was used.

For library construction, adapters were added to total RNA $(150 \mu \mathrm{g} / \mathrm{mL})$ and cDNA was synthesized using a Superscript Double-Stranded cDNA Synthesis kit (Invitrogen). cDNA was enriched through PCR and the small RNA fraction (10-50 nt) was then separated on a denaturing polyacrylamide gel. Libraries were constructed at the BC Cancer Agency's Michael Smith Genome Sciences Centre http://www.bcgsc.ca/ and sequenced with Illumina SBS deep sequencing technology using an Illumina Genome Analyzer IIx http://www.illumina. $\mathrm{com} /$.

\section{Analysis of small RNA sequencing data}

Various quality filters were applied to raw reads data. Sequences having less than 0.6 chastity value (measured by sequencing software) in the first 25 bases were removed. Adapter sequences were then removed with an in-house $\mathrm{C}++$ program; this program removed any sequence fragments larger than 10nt from the original sequence matching the adapter sequence used in the libraries (5'-ATCTCGTATGCCGTCTTCTGCTTG-3') and sequence fragments shorter than 10nt were only removed if they started after the $15^{\text {th }} \mathrm{nt}$ in the original sequence. The program used the EMBOSS wordfinder tool [54] to find adapter fragments (parameters: minimum match score $=70$, alignment width $=6$, gap opening $0.0+$ gap extension 10 or gap opening $500+$ gap extension 10). Sequences shorter than $15 \mathrm{nt}$, as well as low-complexity and low-quality sequences, were then removed using UNIX commands.

Reads were mapped against known snoRNAs, tRNAs and rRNAs obtained from the Rfam Database [55] and then removed. This was done by using Blastn, v. 2.2.21 (parameters:e-value < 0.0001, ungapped, word size $=4$ ) [56]. Unless indicated, these were the standard parameters used for all mapping analysis.

Reads were then mapped to the cassava genome v. 4 (Cassava Genome Project 2010, http://www.phytozome. net/cassava) (parameters: $e$-value $<1 \mathrm{e}-5,100 \%$ identity) and the preliminary version Xanthomonas axonopodis pv. manihotis genome (Xam genome project, unpublished (parameters; e-value $<1 \mathrm{e}-5,100 \%$ identity).

\section{miRNA and pre-miRNA identification}

To identify phylogenetically-conserved miRNAs, reads from both small RNA libraries were mapped to the set of all mature Viridiplantae miRNAs obtained from miRBase release 16, September 2010 [45] and the complete plant miRNA set obtained from the Plant MiRNA Database PMRD, v. September 2010 [22]. Reads having less than two mismatches with a known miRNA were considered conserved miRNAs [38]. Conserved miRNAs (and their expression profiles) were also identified using the miRProf tool from the UEA sRNA toolkit (default parameters) [28]. Reads from both libraries in the 2024 nt size range were mapped on the cassava genome (Cassava Genome Project 2010, http://www.phytozome. net/cassava) to identify possible novel cassava-specific miRNAs.

Pre-miRNA analysis used the adjacent region ( -150 , $+150 \mathrm{nt}$ ) to mapped positions from a read of interest, extracted from the genome using fastacmd [56]. These were then mapped onto annotated cassava genes and regions having large overlaps (> 25\%) with genes were removed from further analysis.

Two main criteria were considered for real pre-miRNAs: structural features identified from predicted foldings and a secondary structure statistical test. Candidate pre-miRNAs were folded with RNAFold from the Vienna RNA package [57] and mFold v. 3.5 [58]. Additional secondary structures for easier visualization were obtained using the RNAfolding utility in the sRNA toolkit [28]. Structures were analyzed with in-house pearl scripts. Real pre-miRNAs were considered if they had less than six mismatches between predicted mature miRNA and miRNA*, few (maximum three) and short (less than 3nt) asymmetric bulges in the structure [38,59]. Secondary structure minimum folding energy (MFE) significance was calculated using a statistical test; 1,000 random sequences were generated for each possible precursor, maintaining the same base composition and dinucleotide frequencies $(k$-let $=2)$ using ushuffle 
[60]. Random structures were then folded using RNAfold [57] and the p-value was calculated as the percentage of random structures having an MFE equal to or lower than the original precursor [61]. Real pre-miRNAs must had $<0.05$ p-value.

Candidate novel pre-miRNAs were analyzed and filtered using miRcheck (default parameters) [29] MIREAP (parameters, $\mathrm{B}=55, \mathrm{a}=19, \mathrm{~b}=24, \mathrm{u}=1,000, \mathrm{e}=-10$ $\mathrm{kcal} / \mathrm{mol}, \mathrm{d}=200, p=7, \mathrm{v}=10, \mathrm{~s}=100, \mathrm{f}=10)$ [31] https://sourceforge.net/projects/mireap/ and miPred (random-forest prediction module not used) [30] to obtain additional prediction support.

\section{miRNA targets prediction}

miRNA targets were searched using a modified miRanda version v. 2.0 September 2008 [33], as previously described to meet plant miRNA:target pairings criteria [62]. Targets were also searched using psRNAtarget, v. December 2010 (default parameters, http://plantgrn. noble.org/psRNATarget/), an update to the miRU software [63]. Targets were searched in cassava coding sequences (Cassava Genome Project 2010, http://www. phytozome.net/cassava).

\section{miRNA, miRNA* and transient siRNAs in silico quantification}

Reads from both libraries were mapped strand-specifically to quantify miRNA and miRNA* expression using Blastn (e-value $<0.0001,100 \%$ identity, $S=1$ for miRNA and $S=2$ for miRNA*). Expression values were assigned for each family instead of each loci due to high similarity in mature miRNA sequences. Expression values were normalized using this formula: matching reads/total reads $\times 1,000,000$.

Reads ranging from 20 to $24 \mathrm{nt}$ from both sRNA libraries were mapped strand-specifically against all identified possible targets for all cassava miRNAs to quantify possible transient siRNAs and mRNA fragments generated by miRNA-mediated cleavage (e-value $<0.0001,100 \%$ identity, $S=1$ for miRNA and $S=2$ for miRNA). Only the $-100 \mathrm{nt},+100 \mathrm{nt}$ region immediate to the predicted miRNA target gene cleavage site was used for mapping to avoid mapping to random RNA fragments not generated by miRNA-cleavage. Expression values were normalized as the number of reads mapping to target/total reads.

\section{Promoter analysis}

The 1,000 nt upstream region of identified cassava premiRNAs, predicted miRNA targets and randomly-chosen cassava genes were extracted for identifying promoters; only complete and high-quality sequences were extracted. Regions having overlaps with genes were delimited to exclude the gene, unless the overlap was longer than $600 \mathrm{nt}$; in this case, the promoter region was not used. TFBS were identified in these regions as described in [6].

Wilcoxon paired tests (two-tailed) were made with $\mathrm{R}$ version v. 2.12.0 ( $\mathrm{R}$ Development Team) comparing TFBS frequencies for each desired group of genes obtained as: Number of genes with a given TFBS/Total of genes evaluated.

\section{Semi-quantitative RT-PCR analysis}

RNA was extracted from leaves and stem tissues from cassava MCOL1522 non-inoculated and inoculated (4 dpi and 8 dpi) with Xam CIO151, grown as described above, to quantify miRNA target expression. After DNAse I (Fermentas) treatment, cDNA was synthesized using First Strand cDNA Synthesis with oligo dTs (Fermentas). cDNA concentration was normalized after PCR using tubuline primers ( $\mathrm{F}=5$ '-GATCCTACTGGGAAGTACATTGG-3', $\mathrm{R}=$ 5'-GATCATTCTCCACCAACTGA-3'). Gene-specific primers for predicted miRNA targets were designed covering the predicted cleavage site. PCRs were performed with 32 cycles a $94^{\circ}$ $\mathrm{C}$ for $30 \mathrm{~s}, 54-60^{\circ} \mathrm{C}$ for $30 \mathrm{~s}$ and $72^{\circ} \mathrm{C}$ for $30 \mathrm{~s}$. The primers used for each target are listed in Additional file 3.

\section{Additional material}

Additional file 1: Cassava pre-miRNAs descriptions. Positions in the genome are based in the Cassava4 version of the genome available at Cassava Genome Project 2010, http://www.phytozome.net/cassava. Folding energies were calculated with Vienna RNAFold.

Additional file 2: Cassava miRNA targets. miRanda predictions for each miRNA family identified in cassava, predictions were made using annotated cds from the Cassava4 version of the genome available at Cassava Genome Project 2010, http://www.phytozome.net/cassava. Descriptions are based on the available annotations for the most similar Arabidopsis or rice gene.

Additional file 3: Primers used for RT-PCR.

\section{Acknowledgements}

This Project was partially financed with support from DIB, quipu No 201010011438 and Colciencias (Contract Number 221-2008). We would like to thank Adriana Bernal and the LAMFU research group for granting us access to the Xam genome draft, Andres Zapata for his suggestions on script development and data analyses and Simon Cortés for his insights into cassava starch biosynthesis.

\section{Authors' contributions}

ALPQ: Prepared plant materials, processed the sRNA libraries data and drafted the manuscript. AQ: Participated in miRNA predictions and data analyses. OU: Participated in miRNA predictions and data analyses. PV: Carried out the semi-quantitative RT-PCR analysis. CL: Conceived the study, participated in its coordination and helped to draft the manuscript. All authors read and approved the final manuscript.

Received: 30 September 2011 Accepted: 23 February 2012

Published: 23 February 2012 


\section{References}

1. Chisholm ST, Coaker G, Day B, Staskawicz BJ: Host-Microbe Interactions: Shaping the Evolution of the Plant Immune Response. Cell 2006, 124:803-814.

2. Jones-Rhoades MW, Bartel DP, Bartel B: MicroRNAS and their regulatory roles in plants. Annu Rev Plant Biol 2006, 57:19-53.

3. Mateos JL, Bologna NG, Palatnik JF: Biogenesis of Plant MicroRNAs. In Non Coding RNAs in Plants RNA technologies. Edited by: Erdmann and Barciszewski. Berlin: Springer; 2011:251-268.

4. German MA, Pillay M, Jeong DH, et al: Global identification of microRNAtarget RNA pairs by parallel analysis of RNA ends. Nat Biotechnol 2008, 26:941-946.

5. Xie Z, Khanna K, Ruan S: Expression of microRNAs and its regulation in plants. Seminars in Cell and Developmental Biology 2010, 21(8):790-7.

6. Megraw M, Hatzigeorgiou AG: MicroRNA promoter analysis. Methods $\mathrm{Mol}$ Biol 2010, 592:149-161

7. Meng Y, Huang F, Shi Q, et al: Genome-wide survey of rice microRNAs and microRNA-target pairs in the root of a novel auxin-resistant mutant. Planta 2009, 230:883-898.

8. Kong WW, Yang ZM: Identification of iron-deficiency responsive microRNA genes and cis-elements in Arabidopsis. Plant Physiol Biochem 2010, 48:153-159.

9. Cui X, Xu SM, Mu DS, Yang ZM: Genomic analysis of rice microRNA promoters and clusters. Gene 2009, 431:61-66.

10. Shukla LI, Chinnusamy V, Sunkar R: The role of microRNAs and other endogenous small RNAs in plant stress responses. BBA - Gene Regulatory Mechanisms 2008, 1779:743-748.

11. Navarro L, Dunoyer $P$, Jay $F$, et al: A plant miRNA contributes to antibacterial resistance by repressing auxin signaling. Science 2006 312:436-439

12. Navarro L, Jay F, Nomura K, He SY, Voinnet O: Suppression of the microRNA pathway by bacterial effector proteins. Science 2008, 321:964-967

13. Zhang W, Gao S, Zhou X, et al: Bacteria-responsive microRNAs regulate plant innate immunity by modulating plant hormone networks. Plant Mol Biol 2010, 75:93-105.

14. Li Y, Zhang QQ, Zhang J, et al: Identification of MicroRNAs involved in pathogen-associated molecular pattern-triggered plant innate immunity. Plant Physiol 2010, 152:2222.

15. Fahlgren N, Howell MD, Kasschau KD, et al: High-throughput sequencing of Arabidopsis microRNAs: evidence for frequent birth and death of MIRNA genes. PLoS One 2007, 2:e219.

16. El-Sharkawy MA: Cassava biology and physiology. Plant Mol Biol 2004, 56:481-501.

17. Ziska LH, Runion GB, Tomecek $M$, et al: An evaluation of cassava, sweet potato and field corn as potential carbohydrate sources for bioethanol production in Alabama and Maryland. Biomass Bioenergy 2009 33:1503-1508.

18. Blagbrough IS, Bayoumi SAL, Rowan MG, Beeching JR: Cassava: an appraisal of its phytochemistry and its biotechnological prospects. Phytochemistry 2010, 71:1940-1951.

19. Verdier $\mathrm{V}$ : Bacteriosis vascular (o añublo bacteriano) de la yuca causada por Xanthomonas axonopodis pv. manihotis. En CIAT eds La yuca en el Tercer Milenio Sistemas modernos de producción procesamiento utilización y comercialización 2002, 148-159.

20. Gregory PJ, Ingram JSI, Brklacich M: Climate change and food security. Philosophical transactions of the royal society of London-series $B$. Biological Sciences 2005, 360:2139-2148.

21. Mhuantong W, Wichadakul D: MicroPC (microPC): a comprehensive resource for predicting and comparing plant microRNAs. BMC Genomics 2009, 10:366.

22. Zhang Z, Yu J, Li D, et al: PMRD: plant microRNA database. Nucleic Acids Res 2010, 38:D806-D813.

23. Amiteye S, Corral JM, Sharbel TF: Overview of the potential of microRNAs and their target gene detection for cassava (Manihot esculenta) improvement. J Biotechnol 2011, 10:2562-2573.

24. Zeng C, Wang W, Zheng Y, et al: Conservation and divergence of microRNAs and their functions in Euphorbiaceous plants. Database 2009, 38:981-995.

25. Griffiths-Jones S: miRBase: microRNA sequences and annotation. Wiley Online Library 2010, Chapter 12: Unit 12.9.1-10.
26. Cassava Genome Project 2010: Cassava Genome Project 2010 [http://www. phytozome.net/cassava].

27. Zhang Z, Yu J, Li D, et al: PMRD: plant microRNA database. Nucleic Acids Res 2010, 38:D806-D813.

28. Moxon S, Schwach F, Dalmay T, et al: A toolkit for analysing large-scale plant small RNA datasets. Bioinformatics 2008, 24:2252-2253.

29. Jones-Rhoades MW, Bartel DP: Computational identification of plant microRNAs and their targets, including a stress-induced miRNA. Mol Cell 2004, 14:787-799.

30. Jiang $P, W u H$, Wang $W$, et al: MiPred: classification of real and pseudo microRNA precursors using random forest prediction model with combined features. Nucleic Acids Res 2007, 35:W339-W344.

31. Chen X, Li Q, Wang J, et al: Identification and characterization of novel amphioxus microRNAs by Solexa sequencing. Genome Biol 2009, 10:R78.

32. Altschul SF, Gish W, Miller W, Myers EW, Lipman DJ: Basic local alignment search tool. I Mol Biol 1990, 215:403-410.

33. Enright AJ, John B, Gaul U, et al: MicroRNA targets in Drosophila. Genome Biol 2003, 5:R1

34. Abdel-Ghany SE, Pilon M: MicroRNA-mediated systemic down-regulation of copper protein expression in response to low copper availability in Arabidopsis. J Biol Chem 2008, 283:15932-15945.

35. Cuperus JT, Fahlgren N, Carrington JC: Evolution and Functional Diversification of MIRNA Genes, the Plant Cell Online 2011, 23:431-442.

36. Kessler SA, Shimosato-Asano H, Keinath NF, et al: Conserved Molecular Components for Pollen Tube Reception and Fungal Invasion. Science 2010, 330:968-971

37. Escobar-Restrepo J-M, Huck N, Kessler S, et al: The FERONIA receptor-like kinase mediates male-female interactions during pollen tube reception. Science 2007, 317:656-660.

38. Meyers BC, Axtell MJ, Bartel B, et al: Criteria for annotation of plant MicroRNAs. Plant Cell 2008, 20:3186-3190

39. Luo QJ, Samanta MP, Köksal F, et al: Evidence for antisense transcription associated with microRNA target mRNAs in Arabidopsis. PLoS Genet 2009 5:e1000457.

40. Megraw M, Baev V, Rusinov V, et al: MicroRNA promoter element discovery in Arabidopsis. RNA 2006, 12(9):1612-1619.

41. Ruiz-Ferrer $V$, Voinnet $O$ : Roles of plant small RNAs in biotic stress responses. Annu Rev Plant Biol 2009, 60:485-510.

42. Katiyar-Agarwal $\mathrm{S}$, Jin $\mathrm{H}$ : Role of small RNAs in host-microbe interactions. Annu Rev Phytopathol 2010, 48:225.

43. Fu J, Liu H, Li Y, et al: Manipulating broad-spectrum disease resistance by suppressing pathogen-induced auxin accumulation in rice. Plant Physiol 2011, 155:589-602.

44. Ding $X, C a o Y$, Huang $L$, et al: Activation of the indole-3-acetic acid-amido synthetase $\mathrm{GH} 3-8$ suppresses expansin expression and promotes salicylate- and jasmonate-independent basal immunity in rice. Plant Cell 2008, 20:228-240.

45. Griffiths-Jones S, Saini HK, Van Dongen S, Enright AJ: miRBase: tools for microRNA genomics. Nucleic Acids Res 2008, 36:D154-D158.

46. Liu H, Tian X, Li Y, Wu C: Microarray-based analysis of stress-regulated microRNAs in Arabidopsis thaliana. RNA 2008, 14:836-843.

47. Sunkar R, Zhu J: Novel and stress-regulated microRNAs and other small RNAs from Arabidopsis. Sci STKE 2004, 16:2001-2019.

48. Borkow G, Gabbay J: Putting copper into action: copper-impregnated products with potent biocidal activities. The FASEB journal official publication of the Federation of American Societies for Experimental Biology 2004, 18:1728-1730.

49. Yuan M, Chu Z, Li X, Xu C, Wang S: The Bacterial Pathogen Xanthomonas oryzae overcomes rice defenses by regulating host copper redistribution. Plant Cell 2010, 22:1-14

50. Axtell MJ, Jan C, Rajagopalan R, Bartel DP: A two-hit trigger for siRNA biogenesis in plants. Cell 2006, 127:565-577.

51. Howell MD, Fahlgren N, Chapman EJ, et al: Genome-wide analysis of the RNA-DEPENDENT RNA POLYMERASE6/DICER-LIKE4 pathway in arabidopsis reveals dependency on miRNA- and tasiRNA-directed targeting[W][OA]. Plant Cell 2007, 19:926-942.

52. Ronemus M, Vaughn MW, Martienssen RA: MicroRNA-targeted and small interfering RNA-mediated mRNA degradation is regulated by argonaute, dicer, and RNA-dependent RNA polymerase in Arabidopsis. Plant Cell $2006,18: 1559-1574$ 
53. Khraiwesh B, Arif MA, Seumel Gl, et al: Transcriptional control of gene expression by microRNAs. Cell 2010, 140:111-122.

54. Rice P, Longden I, Bleasby A: EMBOSS: the European molecular biology open software suite. Trends Genet 2000, 16:276-277.

55. Gardner PP, Daub J, Tate JG, et al: Rfam: updates to the RNA families database. Nucleic Acids Res 2009, 37:D136-D140.

56. Altschul SF, Madden TL, Schaffer AA, et al: Gapped BLAST and PSI-BLAST: a new generation of protein database search programs. Nucleic Acids Res 1997, 25:3389-3402.

57. Hofacker IL, Fontana W, Stadler PF, et al: Fast folding and comparison of RNA secondary structures. Monatshefte für Chemie Chemical Monthly 1994, 125:167-188.

58. Zuker M: Mfold web server for nucleic acid folding and hybridization prediction. Nucleic Acids Res 2003, 31:3406-3415.

59. Ambros $V$, Bartel $B$, Bartel DP, et al: $A$ uniform system for microRNA annotation. RNA 2003, 9:277.

60. Jiang $M$, Anderson J, Gillespie J, Mayne M: uShuffle: a useful tool for shuffling biological sequences while preserving the k-let counts. BMC Bioinforma 2008, 9:192.

61. Freyhult E, Gardner PP, Moulton V: A comparison of RNA folding measures. BMC Bioinforma 2005, 6:241.

62. Pérez-Quintero AL, Neme R, Zapata A, López C: Plant microRNAs and their role in defense against viruses: a bioinformatics approach. BMC Plant Biol 2010, 10:138.

63. Zhang Y: miRU: an automated plant miRNA target prediction server. Gene 2005, 33:701-704.

doi:10.1186/1471-2229-12-29

Cite this article as: Pérez-Quintero et al:: Bioinformatic identification of cassava miRNAs differentially expressed in response to infection by Xanthomonas axonopodis pv. manihotis. BMC Plant Biology 2012 12:29.

\section{Submit your next manuscript to BioMed Central} and take full advantage of:

- Convenient online submission

- Thorough peer review

- No space constraints or color figure charges

- Immediate publication on acceptance

- Inclusion in PubMed, CAS, Scopus and Google Scholar

- Research which is freely available for redistribution

Submit your manuscript at www.biomedcentral.com/submit
Ciomed Central 